\title{
TWO NOTES ON NILPOTENCY AND STANDARD ALGEBRAS
}

\author{
ERNEST L. STITZINGER
}

\begin{abstract}
Two results on nilpotency which are known to hold for Jordan and alternative algebras are shown to hold for standard algebras as well. These results in the known cases arose when developing a Cartan theory for these classes.
\end{abstract}

1. Introduction. If $N$ is a nil subalgebra of a $J$-algebra $A$ and if $[A, A]$ is semicompletely alternative, then a result of Block [2] shows that the enveloping associative algebra $U(A, N)^{*}$ of the set $U(A, N)$ of right and left multiplications of $A$ by elements of $N$ is nilpotent. For standard algebras this result will be sharpened to a result which is known to hold for Jordan and alternative algebras. This is Theorem 1. As an application, a Cartan theory for standard algebras, of characteristic not 3 , paralleling the known ones for Jordan and alternative algebras can be developed using the same operator introduced by Foster [3] and a refinement of the Pierce decomposition [8] using identities in $[9$, p. 205]. This includes a theory of $a$-nilpotence, the existence of Cartan subalgebras except over fields with relatively few elements, a relation with the generic trace and the usual conjugacy theorem using the derivation $D(x, y)=\left[L_{x}+R_{x}, L_{y}+R_{y}\right]$. The standard algebra development follows the known ones closely, hence is not included. An exception is the standard algebra version of a lemma of Schafer which leads to Engel's theorem. This is Theorem 2.

2. The results. A finite dimensional algebra $A$ over a field $\Phi$ of characteristic not 2 is called standard if the following identities hold in $A$

$$
\begin{array}{r}
(w x, y, z)+(x z, y, w)+(w z, y, x)=0 \\
(x, y, z)+(z, x, y)-(x, z, y)=0 \\
\left(x, y, x^{2}\right)=0
\end{array}
$$

where $(r, s, t)$ is the associator $(r, s, t)=(r s) t-r(s t)$. Note that (3) is a consequence of (1) if characteristic $\Phi \neq 3$. Standard algebras are discussed in [1], [7] and [9]. In particular, standard algebras are flexible, Jordan admissible

Received by the editors January 10, 1975 and, in revised form, March 24, 1975.

AMS (MOS) subject classifications (1970). Primary 17A15, 17A30.

(1) American Mathematical Society 1977 
and Lie admissible and the class includes all commutative Jordan algebras as well as associative algebras.

Let $A$ be a standard algebra and let $A^{+}$be the associated Jordan algebra whose space is the same as that of $A$ and multiplication is defined by $a \cdot b=(a b+b a) / 2$. Let $B$ be a subalgebra of $A, U(A, B)$ be the collection of all right and left multiplications of $A$ by elements of $B$ and let $U(A, B)^{*}$ be the enveloping associative algebra of $U(A, B)$. For $x \in B$, let $R_{x}^{+}$be defined by $y R_{x}^{+}=y \cdot x$ for all $y \in A$ and let $d_{x}$ be defined by $y d_{x}=[y, x]=y x-x y$ for all $y \in A$. The identity

$$
c d=\frac{1}{2}[c, d]+c \cdot d
$$

in $A$ yields that a subspace of $A$ which is invariant under $R_{x}^{+}$and $d_{x}$ is also invariant under $R_{x}$ and $L_{x}$. Furthermore Schafer has noted [9, p. 203] that

$$
[x y, z]=[x, z] y+x[y, z]
$$

is an identity in any standard algebra. It follows that $d_{x}$ is a derivation of $A$ and hence of $A^{+}$.

Theorem 1. Let $A$ be a standard algebra and let $B$ be $a$ subalgebra of $A$ and let $N$ be the nilpotent radical of $B$. Then $U(A, N)$ is contained in the radical of $U(A, B)^{*}$.

Proof. Let $R^{+}$be the subalgebra of $U\left(A^{+}, B^{+}\right)^{*}$ generated by all elements of the form $R_{x_{1}}^{+} \cdots R_{x_{n}}^{+}$where at least one of the $x_{i} \in N$. Then $R^{+}$is nilpotent by [5, Theorem 1]. Let $M_{i}=A\left(R^{+}\right)^{i}, i=0, \ldots$ Then for some $m, M_{m}=0$. Note that $\left(R^{+}\right)^{i}$ is generated by all elements of the form $R_{x_{1}}^{+} \cdots R_{x_{n}}^{+}$where at least $i$ of the $x_{1}, \ldots, x_{n}$ are in $N$. Clearly $M_{i} R_{x}^{+} \subseteq M_{i}$ for each $x \in B$. Also $M_{i} d_{x} \subseteq M_{i}$ since $d_{x}$ is a derivation of $A^{+}$. Hence $M_{i}$ is invariant under $U(A, B)$, and $U(A, B)^{*}$ acts on $\bar{M}_{i}=M_{i-1} / M_{i}$. Let $R$ be the ideal of $U(A, B)^{*}$ generated by $U(A, N)$. Then $R$ is generated by $\pi=$ \{products of left and right multiplications of $A$ by elements of $B$ such that at least one of the multiplications is by an element of $N\}$. Let $R_{i}$ and $\pi_{i}$ be, respectively, the sets of linear transformations induced by $R$ and $\pi$ on $\bar{M}_{i}$. To show that $R$ is nilpotent it suffices to prove that each $R_{i}$ is nilpotent. We claim that $M_{i-1} R \subseteq\left[N, M_{i-1}\right]$ $+M_{i}$. By using (4) it follows that $M_{i-1} U(A, N) \subseteq\left[N, M_{i-1}\right]+M_{i}$. Using (4) again,

$$
\begin{aligned}
B\left(\left[N, M_{i-1}\right]+M_{i}\right) & \subseteq\left[B,\left[N, M_{i-1}\right]+M_{i}\right]+B \cdot\left(\left[N, M_{i-1}\right]+M_{i}\right) \\
& \subseteq\left[B,\left[N, M_{i-1}\right]\right]+\left[B, M_{i}\right]+B \cdot\left[N, M_{i-1}\right]+B \cdot M_{i} .
\end{aligned}
$$

The second and fourth terms on the right-hand side are contained in $M_{i}$ since $M_{i}$ is $B$-invariant. Since $A$ is Lie admissible, the Jacobi identity yields that $\left[B,\left[N, M_{i-1}\right]\right] \subseteq\left[N, M_{i-1}\right]$. Now consider $B \cdot\left[N, M_{i-1}\right]$ and let $c \in B, x \in N$ and $w \in M_{i-1}$. Then, using (5), 


$$
\begin{aligned}
c \cdot[x, w] & =\frac{1}{2}(c[x, w]+[x, w] c) \\
& =\frac{1}{2}([c x, w]-[c, w] x+[x c, w]-x[c, w]) \\
& =\frac{1}{2}([c x, w]+[x c, w])-x \cdot[c, w] \in\left[N, M_{i-1}\right]+M_{i} .
\end{aligned}
$$

Hence $D_{i} \equiv\left[N, M_{i-1}\right]+M_{i}$ is invariant under $L_{x}, x \in B$, and similarly it is invariant under $R_{x}, x \in B$. Hence $M_{i-1} R \subseteq D_{i}$ and $D_{i}$ is $B$-invariant by the foregoing. Now to show that $R_{i}$ acts nilpotently on $\bar{M}_{i}$, it suffices to verify that $R_{i}$ acts nilpotently on $\bar{D}_{i}=D_{i} / M_{i}$. Since commutators of $A$ are contained in the nucleus [7, p. 335, (3)],

$$
\bar{D}_{i}\left(\bar{L}_{x} \bar{L}_{y}-\bar{L}_{y x}\right)=0, \quad \bar{D}_{i}\left(\bar{R}_{x} \bar{R}_{y}-\bar{R}_{x y}\right)=0 \quad \text { and } \quad \bar{D}_{i}\left(\bar{R}_{x} \bar{L}_{y}-\bar{L}_{y} \bar{R}_{x}\right)=0
$$

for all $x, y \in B$. Hence each element of $\left\{\pi_{i} \mid \bar{D}_{i}\right\}$ can be written as one of the following: $\bar{L}_{x}, \bar{R}_{x}, \bar{L}_{x} \bar{R}_{y}$ or $\bar{L}_{y} \bar{R}_{x}$ where $x \in N, y \in B$, all of which are nilpotent. Furthermore $\left\{\pi_{i} \mid \bar{D}_{i}\right\}$ is a weakly closed set of linear transformations using the multiplicative semigroup $U(A, B)^{*}$. Hence $\left\{\pi_{i} \mid \bar{D}_{i}\right\}^{*}=R_{i} \mid \bar{D}_{i}$ is nilpotent by Jacobson's refinement to Engel's theorem [4, p. 33] and the proof is complete.

We use the same definitions of $a$-nilpotency as introduced by Foster [3]. Hence for $b_{1}, b_{2}, b_{3} \in A$, let

$$
a\left(b_{1}, b_{2}, b_{3}\right)=b_{3}\left(b_{1} b_{2}\right)+\left(b_{2} b_{1}\right) b_{3}-\left(b_{2} b_{3}\right) b_{1}-b_{1}\left(b_{3} b_{2}\right) .
$$

Since $A$ is standard

$$
a\left(b_{1}, b_{2}, b_{3}\right)=\left[b_{2},\left[b_{1}, b_{3}\right]\right]+2\left(b_{1}, b_{2}, b_{3}\right) .
$$

Let $S\left(b_{2}, b_{3}\right)$ be the linear transformation defined on $A$ by $x S\left(b_{2}, b_{3}\right)$ $=a\left(x, b_{2}, b_{3}\right)$. If $b \in A$, then $b$ is called $a$-nilpotent if $S(b, b)$ is a nilpotent linear transformation. The usual Engel theorem asserts that if each $w \in A$ is $a$-nilpotent, then $A$ is $a$-nilpotent. The standard algebra version follows the usual procedure except for the following

THEOREM 2. Let $A$ be a standard algebra with unity 1 , over a field of characteristic not 3. If $w \in A$ is a-nilpotent, then every $S\left(w^{i}, w^{j}\right), i, j=0,1$, $\ldots$, is nilpotent.

Proof. First note that $S(b, c)$ may be written, using (6) and (7), as

$$
S(b, c)=R_{b} L_{c}+L_{b} R_{c}-L_{b c}-R_{c b}
$$

or

$$
S(b, c)=R_{c} L_{b}-L_{c} L_{b}-R_{c} R_{b}+L_{c} R_{b}+2\left(R_{b} R_{c}-R_{b c}\right) .
$$

Since $w^{0}=1$, the result is clear if $i=0$ or $j=0$. Assume $i, j>0$. Let $A_{w}$ be the associative algebra of all linear transformations of $A$ generated by $R_{w}, L_{w}$, $R_{w^{2}}$ and the identity linear transformation. Then $A_{w}$ contains all $R_{b}$ and $L_{b}$ 
where $b=w^{i}, i=0,1, \ldots$, by $[1, \mathrm{p} .556$, Lemma 5$]$ and $A_{w}$ is commutative by $\left[1\right.$, p. 577, Theorem 5]. Hence the result will follow if every $S\left(w^{i}, w^{j}\right), i, j$ $>0$, has the form $T_{i j} S(w, w)$ where $T_{i j} \in A_{w}$. We show this by induction on $h=i+j$. Since $S\left(w^{i}, w^{j}\right)=S\left(w^{j}, w^{i}\right)$ by (8), it suffices to show that $S\left(w^{i}, w^{j+1}\right)=T_{i, j+1} S(w, w)$ assuming that $S\left(w^{k}, w^{l}\right)=T_{k l} S(w, w)$ for all $k, l$ $>0$ and $k+l \leqslant i+j$. In order to obtain this we show that

$$
S(x, y z)=R_{z} S(x, y)+R_{y} S(x, z)+S(y, z) L_{x}-S(y, z) R_{x}
$$

where $x, y, z$ are nonnegative integral powers of $w$. Now using (8) and (9)

$$
\begin{aligned}
S(x, y z) & -R_{z} S(x, y)-R_{y} S(x, z)-S(y, z) L_{x}+S(y, z) R_{x} \\
= & R_{y z} L_{x}-L_{y z} L_{x}-R_{y z} R_{x}+L_{y z} R_{x}+2\left(R_{x} R_{y z}-R_{x(y z)}\right) \\
& -R_{z}\left(R_{y} L_{x}-L_{y} L_{x}-R_{y} R_{x}+L_{y} R_{x}+2\left(R_{x} R_{y}-R_{x y}\right)\right) \\
& -R_{y}\left(R_{z} L_{x}-L_{z} L_{x}-R_{z} R_{x}+L_{z} R_{x}+2\left(R_{x} R_{z}-R_{x z}\right)\right) \\
& -\left(R_{y} L_{z}+L_{y} R_{z}-L_{y z}-R_{z y}\right) L_{x}+\left(R_{y} L_{z}+L_{y} R_{z}-L_{y z}-R_{z y}\right) R_{x} \\
\equiv & H .
\end{aligned}
$$

All terms with a factor of 2 add together to give 0 by $[9$, p. 205, (12)]. Of the ten terms with a factor of $L_{x}$ on the right, six add together to give 0 , using that $A_{w}$ is commutative. The same is true with $L_{x}$ replaced by $R_{x}$. Hence

$$
\begin{aligned}
H= & R_{y z} L_{x}-R_{z} R_{y} L_{x}-R_{y} R_{z} L_{x}+R_{z y} L_{x}-R_{y z} R_{x} \\
& +R_{z} R_{y} R_{x}+R_{y} R_{z} R_{x}-R_{z y} R_{x} .
\end{aligned}
$$

For any $v \in A, v H=[(v, y, z)+(v, z, y), x]=0$ since each associator in $A$ commutes with each element in $A$ by [7, (7)]. Hence $H=0$. Now

$$
S\left(w^{i}, w^{j} w\right)=R_{w} S\left(w^{i}, w^{j}\right)+R_{w^{j}} S\left(w^{i}, w\right)+S\left(w^{j}, w\right) L_{w^{i}}-S\left(w^{j}, w\right) R_{w^{i}} .
$$

This reduction and the commutativity of $A_{w}$ give the result.

\section{REFERENCES}

1. A. A. Albert, Power-associative rings, Trans. Amer. Math. Soc. 64 (1948), 552-593. MR 10, 349.

2. R. E. Block, $A$ unification of the theories of Jordan and alternative algebras, Amer. J. Math. 94 (1972), 389-412. MR 47 \# 1887.

3. D. M. Foster, On Cartan subalgebras of alternative algebras, Trans. Amer. Math. Soc. 162 (1971), 225-238.

4. N. Jacobson, Lie algebras, Interscience, New York, 1966.

5. Cartan subalgebras of Jordan algebras, Nagoya Math. J. 27 (1966), 591-609. MR 34 \#218. 
6. - Structure and representations of Jordan algebras, Amer. Math. Soc. Colloq. Publ., vol. 39, Amer. Math. Soc., Providence, R.I., 1968. MR 40 \# 4330.

7. E. Kleinfeld, Standard and accessible rings, Canad. J. Math. 8(1956), 335-340. MR 17, 1180.

8. K. McCrimmon, Structure and representations of noncommutative Jordan algebras, Trans. Amer. Math. Soc. 121 (1966), 187-199. MR 32 \#5700.

9. R. D. Schafer, Standard algebras, Pacific J. Math. 29 (1969), 203-223. MR 39 \#5647.

Department of Mathematics, North Carolina State University, Raleigh, North CaroLINA 27607 